\title{
ZWEISPRACHIGE SONGS. SPRACHMUSTER TRANSKULTURELLER INSZENIERUNGEN
}

\author{
Eckhard John
}

Als Paul McCartney im Jahr 2010 den »Gershwin Prize for Popular Song « der Library of Congress in Washington verliehen bekam, reizte inn die Aufführung eines Songs bei den Feierlichkeiten im Weißen Haus am 2. Juni ganz besonders:

»Michelle, ma belle,

These are words that go together well,

My Michelle.

Michelle, ma belle,

Sont les mots qui vont très bien ensemble,

Très bien ensemble.

I love you, I love you, I love you

That's all I want to say«

(The Beatles: »Michelle«, 1965).

Als dieses Lied auf dem Beatles-Album Rubber Soul erschien, ahnte noch niemand die Weltkarriere eines Songs, der sicherlich nicht nur im Hause Obama innerlich unmittelbar mitklingt. ${ }^{1}$ »Michelle « kann als Beispiel dafür stehen, was im Folgenden unter zweisprachigen Songs verstanden wird: Lieder, in denen zwei Sprachen gleichzeitig in einem Text verwendet werden. Demgegenüber spielen Songs, die in verschiedenen Sprachen existieren (als

1 »Michelle« gilt (nach »Yesterday«) als das am zweithäufigsten gespielte Lied der Beatles. Als Single war »Michelle 1966 in der Aufnahme der Overlanders ein Nr. 1-Hit in Großbritannien, während in den US-Charts die Version des britischen Duos David \& Jonathan erfolgreich war. 1967 mit dem Grammy Award für den »Song of the Year « ausgezeichnet, wurde »Michelle« 1999 von BMI (Broadcast Music, Inc.) als Nr. 42 unter den 100 am häufigsten aufgeführten Songs des 20. Jahrhunderts gelistet; vgl. BMI Announces Top 100 Songs of the Century (13.12.1999), http://www.bmi.com/news/entry/232893 (letzter Zugriff 15.7. 2015). 
Übersetzungen oder Umdichtungen) hier keine Rolle, auch nicht Lieder von Interpreten, die verschiedene Stücke in verschiedenen Sprachen singen.

Zur Verortung meines Beitrags möchte ich vorausschicken, dass es sich um einen ersten Aufriss zum Thema handelt, um Einblicke in die Werkstatt der Recherche und Aussichten auf das Ausgrabungsfeld mit seiner Fülle an Material. Der Analyse en detail geht der Schritt des Sammelns und Sortierens voran. Und so zielt mein Beitrag primär auf eine Kartographie der bilingualen Liedspuren, auf die Erstellung eines ersten Orientierungsrasters zur Präsenz und Funktion zweisprachiger Songs. Hinzu kommt, dass ich kein Sprachwissenschaftler oder Linguist bin, und schon gar kein Spezialist für Zweisprachigkeit - somit wird mein Beitrag auch keine entsprechenden Analysen der text- und sprachimmanenten Ebenen oder der Strategien und Finessen des Code-Switching bieten. Ich nähere mich der Thematik vielmehr als Beobachter von außen, als »Liebhaber « sozusagen, der sich als ambitionierter Amateur ohne langjähriges Spezialtraining in Sachen Bilingualität einfach mal auf den Platz wagt; »Liebhaber « freilich auch in dem Sinne, dass ich irgendwann gemerkt habe, dass mir Bands gefallen, die mit zweisprachigen Texten arbeiten. ${ }^{2}$

Ausgangspunkt meiner Recherchen war zunächst der Eindruck, dass die zunehmende Etablierung bilingualer Songs in der populären Musik ein vergleichsweise junges Phänomen ist, das sich in den letzten 20 bis 30 Jahren entwickelt hat. Es hat sich parallel zu den Entwicklungen des Musikmarktes (im Zeichen von »world music « und »Weltmusik«) herausgebildet, scheint aber auch eine Reaktion auf allgemeine Prozesse der Globalisierung, Migration und neuer Regionalität sowie den verschiedenen Wechselwirkungen zwischen diesen drei Faktoren zu sein. Gerade in diesen Kontexten gehen zweisprachige Songs häufig mit einem emanzipatorischen Anspruch von Minderheiten einher, zumal in einem politischen Sinne. An solche Eindrücke knüpften meine Fragen zunächst an, simple und elementare Fragen wie: Lassen sich diese Impressionen verallgemeinern? Wie sieht die Situation insgesamt aus? Wie ist sie zu verstehen und zu interpretieren?

Ein zweiter Ansatzpunkt meiner Überlegungen ist die Beobachtung, dass zweisprachige Songs oft gar nicht bewusst als solche erinnert werden. Dies habe ich im Zuge meiner Recherchen immer wieder gemerkt, wenn ich Freunde, Bekannte oder auch Kollegen gefragt habe: Welche zweisprachigen Songs kennst du? In der Regel war da zunächst eine komplette Leerstelle:

2 Mit Blick auf Dietrich Helms' Plädoyer für eine Kenntlichmachung des »Ichs « in der Popularmusikforschung (Helms 2014) bekenne ich gerne, dass für mich beispielsweise die ersten beiden Platten von Mano Negra (1988/89) in dieser Hinsicht eine wichtige Rolle gespielt haben. 
meist fiel ihnen dazu spontan gar nichts ein - nicht einmal international und generationenübergreifend bekannte Songs (wie das eingangs erwähnte »Michelle«). Weit präsenter waren demgegenüber Musiker oder Bands, in deren Repertoire unterschiedliche Songs in verschiedenen Sprachen vertreten sind. Das änderte sich erst im Rahmen längerer Gespräche über dieses Sujet oder mit deutlicher Verzögerung: manchmal bekam ich erst Tage oder Wochen später Hinweise auf zweisprachige Songs, die den Befragten doch noch eingefallen waren (oder die sie mittlerweile gehört hatten). Als Erinnerungsfaktor spielt die Zweisprachigkeit von Songs demnach offenbar keine große Rolle - aber es gibt solche Songs, und es gibt davon mehr als man auf Anhieb meint. Der Umstand, dass Mehrsprachigkeit in der Regel kein Kriterium der Erinnerung an diese Songs ist, sollte nicht dazu verleiten, sie als vernachlässigbares Randphänomen einzuschätzen. Er macht es nur aufwändiger, das Material zusammenzutragen. ${ }^{3}$

Ein dritter Ausgangspunkt meines Interesses waren eigene Untersuchungen zur Zweisprachigkeit von traditionellen Liedern. Seit ich bemerkt habe, dass diese Lieder in der herkömmlichen »Volkslied«-Forschung ein nahezu unbeachtetes, tendenziell tabuisiertes Feld waren, hat mich diese Thematik interessiert. Als vermeintliche Randerscheinung hat man sie dort nur selten zur Kenntnis genommen, meist im Kontext der »makkaronischen Poesie « und als Spielart humoristischer Lieder. Auch die archivalische Präsenz mehrsprachiger Lieder in den einschlägigen »Volkslied«-Sammlungen ist äußerst begrenzt und überschaubar. Denn aufgrund der vielfach nationalistisch aufgeladenen Parameter der Volkslied-Idee wurden mehrsprachige Lieder ehedem eher als störende sprachliche Verunreinigungen denn als willkommene Repräsentanten eines nationalen Liedgutes angesehen, und dementsprechend selten sind sie von den früheren »Volkslied«-Sammlern aufgezeichnet worden. Die vergleichsweise spärliche Archivlage zu solchen Liedern spiegelt somit aber lediglich die selektive Sammelpraxis, nicht jedoch die tatsächliche Existenz solcher Lieder. Demgegenüber lässt sich zeigen, dass sich weit mehr zweisprachige Lieder im traditionellen Repertoire nachweisen lassen, sobald ohne ideologisch bedingte Scheuklappen gearbeitet wird (John 2015). Grob vereinfacht kann man resümieren, dass überall dort, wo unterschiedliche Sprachräume aufeinander treffen, es auch zweisprachige Lieder gegeben hat. Dies betrifft sowohl geographische wie soziale Räume.

3 Mit etwas Ausdauer kommt aber doch eine ganze Menge zusammen - weit mehr jedenfalls, als ich hier im Einzelnen erwähnen oder vorstellen könnte. Meine Liste umfasst bislang über 250 Songs (und selbst diese bilden sicher nur die berühmte Spitze des Eisbergs). 
In geographischer Hinsicht sind es: alle Grenzgebiete von Sprachkulturen, ${ }^{4}$ Regionen mit sprachlichen Minderheiten (z.B. die Sorben) oder multikulturelle Regionen (wie Südosteuropa). In sozialer Hinsicht sind Bildung und Mobilität die entscheidenden Faktoren. Das Milieu der Gebildeten war die Heimat der »makkaronischen Poesie«, vor allem bei Klerus und Studenten: die studentischen Kommersliederbücher enthielten beliebte deutsch-lateinische Mischlieder und bis heute kennt man entsprechende Kirchenlieder (»In dulci jubilo, nun singt und seid froh«). Mobilität spielt besonders in Hinblick auf die Arbeitswelten eine Rolle, zumal bei Berufsgruppen, die buchstäblich Grenzgänger waren, wie Seeleute (Shanties) oder Wanderarbeiter/Gastarbeiter (so genannte »Italienerlieder«), aber - mit fließenden Übergängen dazu - auch ganz allgemein im Kontext von Migration und Einwanderung. Mit anderen Worten: überall dort, wo zweisprachige Kompetenzen bei Menschen vorhanden waren, gab es auch zweisprachige Lieder.

Einige Charakteristika aus dem Bereich der traditionellen Lieder haben auch in der jüngeren populären Musik Geltung: insbesondere hinsichtlich der geographischen Aspekte von Sprachgrenzen, Sprachminoritäten und Einwanderersprachen. Greifen wir die Sprachkombination Englisch und Französisch von »Michelle « nochmal auf, so finden sich unter den Songs, die diese beiden Sprachen verwenden, beispielsweise etliche Songs kanadischer Musiker - ein Faktum, das mit den Gegebenheiten vor Ort korrespondiert und angesichts eines Anteils von ca. $24 \%$ frankophonen Kanadiern wenig überrascht:

\begin{tabular}{|c|c|}
\hline Leonard Cohen & »The Partisan« (Songs From A Room, 1969) \\
\hline Gordon Lightfoot & »Nous Vivons Ensemble« (Summer Side Of Life, 1971) \\
\hline Buffy Sainte-Marie & $\begin{array}{l}\text { "Song Of The French Partisan« (She Used To Wanna Be } \\
\text { A Ballerina, 1971) }\end{array}$ \\
\hline Gilles Vigneault & »I Went To The Market« (J'ai planté un chêne, 1976) \\
\hline Rush & »Circumstances« (Hemispheres, 1978) \\
\hline Daniel Lanois & »Jolie Louise« (Acadie, 1989) \\
\hline Daniel Lanois & »Under A Stormy Sky« (Acadie, 1989) \\
\hline Rufus Wainwright & »Rebel Prince« (Poses, 2001) \\
\hline Arcade Fire & »Une Année Sans Lumière« (Funeral, 2004) \\
\hline Arcade Fire & »Haiti« (Funeral, 2004) \\
\hline Metric & »Poster Of A Girl« (Live It Out, 2005) \\
\hline
\end{tabular}

4 Nimmt man den deutschsprachigen Raum als Beispiel, so wird unmittelbar anschaulich, dass solche Sprachgrenzen wie eine Art Gürtel diesen Raum umgeben und in unterschiedlichsten Kombinationen zweisprachige Lieder hervorbringen (mit Dänisch, Polnisch, Tschechisch, Ungarisch, Slowenisch, Italienisch, Französisch, Flämisch und etlichen weiteren Sprachen). 


\begin{tabular}{|l|l|}
\hline Dany Bédar \& Joel Kroeker & »Déjà Vu« (2007) \\
\hline Arcade Fire & »Black Wave / Bad Vibrations« (Neon Bible, 2007) \\
\hline Arcade Fire & »Black Mirror $($ Neon Bible, 2007$)$ \\
\hline Arcade Fire & »Reflektor (Reflektor, 2013) \\
\hline Arcade Fire & »Joan Of Arc« (Reflektor, 2013) \\
\hline Arcade Fire & »It's Never Over« (Hey Orpheus) (Reflektor, 2013) \\
\hline
\end{tabular}

Tabelle 1: Kanada

Ein anderes Beispiel ist die Cajun-Musik im Süden der USA (zumal in Louisiana), deren Songs vom Französisch des Cajun-County geprägt sind - auch hier liegen englisch-französische Songs nicht nur auf der Hand, sondern sozusagen unmittelbar auf der Zunge:

\begin{tabular}{|c|c|}
\hline Oklahoma Tornadoes & »Dans la prison« (1947) \\
\hline Bobby Page \& The Riff Raffs & »Hippy Ti Yo« (1958) \\
\hline Gene King & »Little Cajun Girl« (1961) \\
\hline Rod Bernard & »Colinda« (1962) \\
\hline Clifton Chenier & »Eh, Petite Fille« (Louisiana Blues \& Zydeco, 1965) \\
\hline Clifton Chenier & »I'm A Hog For You Baby« (Out West, 1974) \\
\hline Clifton Chenier & »Zydeco Two Step« (Hot Pepper, 1980) \\
\hline Jimmy C. Newman & »Big Mamou« (Progressive Country, 1977) \\
\hline $\begin{array}{l}\text { Wayne Toups \& The Crowley } \\
\text { Aces }\end{array}$ & »Bosco Blues« (Cajun Paradise, 1979) \\
\hline Johnnie Allan & »Little Fat Man«(Cajun Country, 1980) \\
\hline Johnnie Allan & »Let's Go Get Drunk« (Cajun Country, 1980) \\
\hline Johnnie Allan & $\begin{array}{l}\text { „Today I Started Loving You Again« (Cajun Country, } \\
\text { 1980) }\end{array}$ \\
\hline Johnnie Allan & »You Win Again« (Cajun Country, 1980) \\
\hline Wayne Toups \& Zydecajun & $\begin{array}{l}\text { "Sweet Joline« (Single } 1988 \text { / Blast From The Bayou, } \\
\text { 1989) }\end{array}$ \\
\hline Wayne Toups \& Zydecajun & »Two-Step Mamou« (Blast From The Bayou, 1989) \\
\hline Wayne Toups \& Zydecajun & »Johnny Can't Dance« (Blast From The Bayou, 1989) \\
\hline Wayne Toups \& Zydecajun & $\begin{array}{l}\text { „Going Back To Big Mamou« (Blast From The Bayou, } \\
\text { 1989) }\end{array}$ \\
\hline Queen Ida \& Her Zydeko Band & »C'est Moi« (Cookin' With Queen Ida, 1989) \\
\hline
\end{tabular}

Tabelle 2: Cajun/Zydeco

Die USA mit ihren ausgeprägten Immigrantenkulturen sind natürlich generell ein immenses Reservoir für zweisprachige Songs, sei es Jiddisch, Italienisch, Polnisch oder das südafrikanische Xhosa (s. Tabelle 3), wobei freilich der Anteil von spanisch-englischen Liedern die zweifellos dominante Sprachkom- 
Eckhard John

\begin{tabular}{|c|c|c|}
\hline $\begin{array}{l}\text { The Barry Sisters } \\
\text { (with Sam Medoff \& } \\
\text { The Yiddish Swingtet) }\end{array}$ & »Oh Mama, I'm So In Love« (1940) & engl. / jiddish \\
\hline $\begin{array}{l}\text { The Barry Sisters } \\
\text { (with Sam Medoff \& } \\
\text { The Yiddish Swingtet) }\end{array}$ & $»$ Yidel Mitn Fiedel « (1946) & engl. / jiddisch \\
\hline Leo Fuld & »Wo ahin soll ich geh'n« (1947) & engl. / jiddisch \\
\hline Louis Prima & »Angelina« (1944 / 1957) & engl. / ital. \\
\hline Louis Prima & »The Manuelo Tarantel« (1949) & engl. / ital. \\
\hline Louis Prima & »Oh Marie« (The Wildest, 1956) & engl. / ital. \\
\hline Miriam Makeba & »Pata Pata« (Pata Pata, 1967) & xhosa / engl. \\
\hline Bobby Vinton & »My Melody Of Love« (1974) & engl. / polnisch \\
\hline Ashia \& The Bison Rouge & $\begin{array}{l}\text { "Shepherd's Wings« (Diesel vs. Lungs, } \\
\text { 2013) }\end{array}$ & engl. / polnisch \\
\hline Ashia \& The Bison Rouge & $\begin{array}{l}\text { »Spirit Dances Evermore« (Diesel vs. } \\
\text { Lungs) }\end{array}$ & engl. / polnisch \\
\hline Gogol Bordello & »Dogs Were Barking« (Gypsy Punks, 2005) & engl. / russisch \\
\hline Gogol Bordello & $\begin{array}{l}\text { „Start Wearing Purple« (Gypsy Punks, } \\
\text { 2005) }\end{array}$ & engl. / russisch \\
\hline Gogol Bordello & »Undestructable« (Gypsy Punks, 2005) & engl. / russisch \\
\hline
\end{tabular}

Tabelle 3: US-Immigratenkulturen

\begin{tabular}{|l|l|}
\hline José Feliciano & "Feliz Navidad« (Feliz Navidad, 1970) \\
\hline Freddy Fender & »Before The Next Teardrop Falls« (1975) \\
\hline Freddy Fender & "Secret Love« (1975) \\
\hline Marty Robbins & "Buenos Dias Argentina« (All Around Cowboy, 1979) \\
\hline Sandi Patty & "Via Dolorosa (Songs From The Heart, 1984) \\
\hline Pete Seeger & "Somos El Barco (We Are The Boat)« (1985) \\
\hline Mellow Man Ace & "Mentirosa« (Escape From Havana, 1989) \\
\hline Texas Tornados & »Hey Baby, Que Paso?« (Texas Tornados, 1990) \\
\hline Texas Tornados & "Soy de San Luis« (Texas Tornados, 1990) \\
\hline Gerardo Mejía & "Rico Suave« (Mo' Ritmo, 1991) \\
\hline Cypress Hill & "Latin Lingo« (Cypress Hill, 1991) \\
\hline Mellow Man Ace & "Brother With Two Tongues« (The Brother With Two Tongues, 1992) \\
\hline Sublime & "Caress Me Down« (Sublime, 1996) \\
\hline Mars Volta & "L'Via L'Viaquez« (Frances The Mute, 2005) \\
\hline Calexico & "Roka (Danza de la Muerte) (Garden Ruin, 2006) \\
\hline Calexico & "Victor Jara's Hands« (Carried To Dust, 2008) \\
\hline
\end{tabular}

Tabelle 4: Englisch - Spanisch (USA) 
bination ist (s. Tabelle 4): von Tex-Mex (Texas Tornados) und Country (Freddy Fender, Marty Robbins) über Folk (Pete Seeger), Pop (José Feliciano), Sacro-Pop (Sandi Patty), Hard Rock (Mars Volta), Indie-Rock (Calexico), Reggae (Sublime) bis hin zum kalifornischen HipHop (Cypress Hill, Mellow Man Ace, Gerardo Mejía).

Auch traditionelle Sprachminoritäten finden Ausdruck in zweisprachigen Songs, zumal im Folk und Folk-Rock: Gälisch und Walisisch in Großbritannien und Irland (s. Tabelle 5), Bretonisch, Korsisch und Okzitanisch in Frankreich. Dort spiegeln sich auch die anderen beiden Sprachraum-Parameter in bilingualen Songs (s. Tabelle 6): Sprachgrenzen (zu Flämisch, Spanisch, Italienisch) und Einwandererkulturen (Französisch-Arabisch).

\begin{tabular}{|l|l|l|}
\hline Clancy Brothers & $\begin{array}{l}\text { „Cruiscín Lán« (Come Fill Your Glass With Us, } \\
\text { 1959) }\end{array}$ & $\begin{array}{l}\text { engl. / irisch- } \\
\text { gälisch }\end{array}$ \\
\hline Altan & "Ta Mo Chleamhnas a Dheanamh« (Altan, 1987) & $\begin{array}{l}\text { engl. / irisch- } \\
\text { gälisch }\end{array}$ \\
\hline Chieftains & »Iimmy, Mó Mhíle Stór« (Tears Of Stone, 1999) & $\begin{array}{l}\text { engl. / irisch- } \\
\text { gälisch }\end{array}$ \\
\hline Silly Wizard & "Fhear A Bhata« (Caledonia's Hardy Sons, 1978) & $\begin{array}{l}\text { engl. / schott.- } \\
\text { gälisch }\end{array}$ \\
\hline Runrig & "Skye« (Heartland, 1985) & $\begin{array}{l}\text { engl. / schott.- } \\
\text { gälisch }\end{array}$ \\
\hline $\begin{array}{l}\text { Gorky's Zygotic } \\
\text { Mynci }\end{array}$ & »Patio Song« (1996) & engl. / walisisch \\
\hline Here Be Dragons & "Hapus lawn« (Bright New Tomorrow, 2007) & engl. / walisisch \\
\hline
\end{tabular}

Tabelle 5: Gälisch / Walisisch

\begin{tabular}{|l|l|l|}
\hline Tri Yann & "Complainte de Yuna Madalen« (1983) & franz. / bretonisch \\
\hline I Muvrini & "Jalàlàbàd« (Umani 2002) & franz. / korsisch \\
\hline $\begin{array}{l}\text { Massilia Sound } \\
\text { System }\end{array}$ & »Au Marché du Soleil« (Òai e libertat, 2007) & franz. / okzitanisch \\
\hline Jacques Brel & »Marieke« (Jacques Brel 5, 1961) & franz. / flämisch \\
\hline Jonatan Cerrada & »Ruban Noir« (La Preuve du Contraire, 2005) & franz. / spanisch \\
\hline Jonatan Cerrada & "Amore« (La Preuve du Contraire, 2005) & franz. / italienisch \\
\hline Cheb Khaled & "Ä̈cha« (Sahra, 1996) & franz. / arabisch \\
\hline
\end{tabular}

Tabelle 6: Frankreich

Ein zweiter Punkt, bei dem sich das Thema Zweisprachigkeit im Bereich traditioneller Lieder mit dem Feld der jüngeren Popularmusik berührt, ist die Dominanz von Einzelsprachen. Diese gründet weniger auf individuellen Faktoren (Muttersprache) als auf dem sozialen Rahmen in dem Sprache benutzt wird (als Amtssprache, vermittelt durch Instanzen wie Schule und Medien, 
modelliert durch Wertzuschreibungen und Normierungen etc.). Schon die national (und nationalistisch) orientierte »Volkslied «-Idee mit ihren dementsprechenden Sprachpräferenzen korrespondierte solchen gesellschaftlichen und politischen Faktoren - aber auch das Musikbusiness mit seinen ökonomischen Interessen und Strategien ist davon nicht unabhängig: Der Markt der populären Musik wurde in dem von Nationalismen aufgeladenen 20. Jahrhundert ebenfalls von dominanten Sprachen beherrscht und die Mehrheit aller Songs ist in einer einzigen Sprache getextet. Es ist somit kein Zufall, dass »Michelle « nahezu der einzige zweisprachige Song im Repertoire der Beatles ist. ${ }^{5}$ Zweisprachige Songs waren lange Zeit eine Ausnahme (und nicht die Regel). Und es war auch eher die Ausnahme, dass Freddie Fender mit seiner spanisch-englischen Version von »Before The Next Teardrop Falls« (1974) einen Nr.1-Hit in den Billboard-Charts und sogar in den CountryCharts landete. Die Regel im Business der kommerziell erfolgreichen Songs waren Songtexte in einer Sprache. Dementsprechend modelliert sind auch unsere Hörgewohnheiten. Dies scheint mir ein wichtiger Grund dafür zu sein, dass Zweisprachigkeit beim Hören zwar sicherlich als solche wahrgenommen wird (bewusst oder unbewusst), dass sie sich aber meist nicht in der Erinnerung festsetzt.

Anderssprachige Elemente in populären Songs dienten lange Zeit primär nur als illustratives Symbol oder Signal, als sprachliches Kolorit für andere Kulturen und fremde Länder. Auch in »Michelle « fungierten die französischen Wendungen in diesem koloristischen Sinne: als Referenz an das damalige Faible für moderne französische Kultur und Bohème. In ähnlicher Weise untermalen die spanischen Einsprengsel im Refrain von Bob Dylans »Romance In Durango (Desire, 1976) das mexikanische Ambiente des im Liedtext evozierten Schauplatzes. In Dylans Oeuvre ist dieser zweisprachige Song ebenfalls singulär. Die Dominanz einer Sprache in den kommerziell erfolgreichen Songs zeigt sich in den 1950er bis 1970er Jahren auch daran, dass sich der koloristische Einsatz anderssprachiger Elemente häufig auf nur wenige prägnante Worte beschränkte - zumal an exponierten Stellen und im Refrain: »Buona sera, signorina kiss me goodnight « (Louis Prima: »Buona Sera«, 1956), »Voulez-vous coucher avec moi?« (Labelle: »Lady Marmelade«, 1974), »Qué Será, Será« (Doris Day, 1957), »Fraulein« (Bobby Helms, 1957), "Vaya Con Dios« (Les Paul \& Mary Ford, 1957), "Chanson d'Amour (Art \& Dotty Todd, 1958; Manhatten Transfer, 1977), »C'est La Vie« (Emerson, Lake

5 Demgegenüber sind »Sun King « (Abbey Road, 1969), mit seinem Fantasie-Spanisch, und »Across The Universe (Let It Be, 1970), mit dem Sanskrit-Einsprengsel »Jai Guru Deva $(\mathrm{Om})$ «, weit weniger prominent und als speziellere Fälle mehr am Rande erwähnenswert. 
\& Palmer, 1977) oder »Voulez Vous« (Abba, 1979). Gleichermaßen gilt dies auch für deutsche Schlager. ${ }^{6}$

Diese koloristische Funktion einer zweiten Sprache in populären Songs hat sich bis in die Gegenwart erhalten. ${ }^{7}$ Doch ihre Formen haben sich seit den 1980er Jahren stark verändert. Mit der Etablierung von Musikvideos werden solche koloristischen Bezüge zweisprachiger Songs auch über entsprechende filmische Inszenierungen hergestellt (z.B. Kylie Minogue: »Je Ne Sais Pas Pourquoi «, 1988). Zudem wurden die Textanteile der zweiten dabei verwendeten Sprache teilweise deutlich umfangreicher, wie »Carbonara« von Spliff beispielhaft zeigt:

»Io voglio viaggiare in Italia

In paese dei limoni.

Brigade Rosse e la Mafia

Cacciano sulla Strada del Sol.

Distruzione della Lira,

Gelati Motta con brio«

(Spliff: »Carbonara«, 1982).

Hier ist der gesamte erste Teil des Songs in (deutschem) Pidgin-Italienisch gehalten und erst nach dem Refrain geht es auf Deutsch weiter, mit gelegentlichen Einlagen von Code-Switching:

»Scusi signorina, willst du auch'n Spliff?

Oder stehst du nur auf Männer mit Schlips?

Ich hab' sonst nichts was ich dir geben kann,

Aber blond bin ich, ist das vielleicht nichts?

$[\ldots]$

Belladonna, ich lad' dich jetzt zum Essen ein,

Mangiare - tu capito? Andiamo!«

(ebd.).

Gleichzeitig kam ein inhaltlicher Aspekt hinzu: Zweisprachigkeit als Kolorit wurde nun auch als Ausdruck politischer Couleur und Parteinahme verwen-

6 Stellvertretend für zahlreiche mögliche Beispiele: »Merci Chérie« (Udo Jürgens, 1966), »Memories Of Heidelberg « (Peggy March, 1967), »Fiesta Mexicana« (Rex Gildo, 1972), »Love Me« (Chris Roberts, 1972), »Eviva Espana« (Imca Marina, 1972).

7 Beispielsweise von der Neuen Deutschen Welle (Ideal: »Monotonie«, 1981; Spliff: »Carbonara«, 1982), über Madonna (»La Isla Bonita«, 1987; »Who's That Girl«, 1987; »|saac«, 2005), bis hin zur letzten Fußball-Weltmeisterschaft (Pitbull: »We Are One «, 2014). 
det, etwa von The Clash mittels eines spanischen Refrains zum englischen Text der »Spanish Bombs «:

»Spanish songs in Andalucia, the shooting sites in the days of ' 39.

Oh, please leave the vendanna open,

Frederico Lorca dead and gone,

Bullet holes in the cemetery walls, the black cars of the Guardia Civil, Spanish bombs on the Costa Rica, I'm flying in on a D.C.10 tonight ...

Spanish bombs, yo t'quierro y finito, yo te querda, oh ma côrazon,

Spanish bombs, yo t'quierro y finito

yo te querda, oh ma côrazon«

(The Clash: »Spanish Bombs «, 1979). ${ }^{8}$

Sting verwendete in »They Dance Alone (Nothing Like The Sun, 1987) ebenfalls eine spanische Textpassage, um seine Klage über die Opfer der Pinochet-Diktatur in Chile mit dem Klang ihrer Sprache zu verbinden. Freilich sind es auch im politischen Kontext häufig nur einzelne oder wenige Worte, die als Symbole der Botschaft oder als Signale der Provokation eingesetzt werden, etwa »Blitzkrieg Bop« (The Ramones, 1976), »California Über Alles« (Dead Kennedys, 1979) ${ }^{9}$ oder »Arbeit Macht Frei« (The Libertines, 2004).

Neben der koloristischen Funktion nimmt Zweisprachigkeit in populären Songs häufig eine übersetzende Funktion ein. Auch dies begleitet die populäre Musik schon seit früher Zeit: »Bei Mir Bist Du Schön«, der erste große Hit der Andrews Sisters 1938, war eine ins Englische transformierte Adaption einer jiddischen Musical-Nummer (wobei vom jiddischen Original nur mehr die ins Deutsche gewendete Refrainzeile übrigblieb). Zugleich spielt dieser Songtext aber auch mit der Szenerie einer beziehungsreichen Übersetzungssituation:

»Bei mir bist du schön, please let me explain,

Bei mir bist du schön means you're grand.

Bei mir bist du schön, again I'll explain,

It means you're the fairest in the land.

8 Die Schreibweise der spanischen Verse folgt der inneren Plattenhülle; sprachlich korrekter wäre: »Yo te quiero infinito / Yo te quiero, oh mi corazón«; ebenso »ventana« (statt: vendanna).

9 Die New Yorker Band Hasidic New Wave machte daraus 1998 - in Anspielung auf den damaligen Bürgermeister der Stadt - »Giuliani Über Alles«. 
I could say bella, bella, even say wunderbar,

Each language only helps me tell you how grand you are«

(The Andrews Sisters: »Bei Mir Bist Du Schön«, 1938).

Das Beispiel der Andrews Sisters verweist auf zwei Aspekte, die für unseren Zusammenhang wesentlich sind. Gemeinsam mit dem funktionellen Gesichtspunkt der Übersetzungsformen kommt hier auch die Herkunft des Materials ins Spiel: Denn Coverversionen sind ebenfalls eine beständige Spielart bilingualer Songs. Häufig dienen bekannte Hits als Vorlage, aber auch Filmschlager, traditionelle und politische Lieder finden Verwendung. Manchmal geht mit berühmten Titeln - wie »Ne me quitte pas « - ein ganzes Bündel an zweisprachigen Coverversionen einher:

\begin{tabular}{|l|l|l|}
\hline Dusty Springfield & "If You Go Away« (The Look Of Love, 1967) & engl. / franz. \\
\hline Lana Cantrell & $\begin{array}{l}\text { »If You Go Away« (And Then There Was Lana, } \\
\text { 1967) }\end{array}$ & engl. / franz. \\
\hline Barbra Streisand & "If You Go Away« (Love Is the Answer, 2009) & engl. / franz. \\
\hline Regina Spektor & $\begin{array}{l}\text { »Don't Leave Me« (What We Saw From The } \\
\text { Cheap Seats, 2012) }\end{array}$ & engl. / franz. \\
\hline Caro Emerald & »If You Go Away« (2013) & engl. / franz. \\
\hline Musica Nuda & "Non andare via« (Musica Nuda 2, 2006) & ital. / franz. \\
\hline Das Blaue Einhorn & »Ne me quitte раз« (Verkauf dein Pferd, 2007) & deutsch / franz. \\
\hline Regina Spektor & »Не Покидай Меня [Ne Pokidai Menja]« (2012) & russisch / franz. \\
\hline
\end{tabular}

Tabelle 7: »Ne me quitte pas« - zweisprachige Cover

Andererseits gibt es auch Adaptionen, die erst in ihrer zweisprachigen Version zu internationalen Hits wurden, wie »Wooden Heart « (Elvis Presley, 1960) oder »Denis « (Blondie, 1978). ${ }^{10}$ Beide Aspekte (Cover und Übersetzung) finden sich häufig unabhängig voneinander, können aber auch in einem Stück zusammentreffen. Bobby Pages Cajun-Song »Hippy Ti Yo« (1958) griff beispielsweise ein Traditional auf und übersetzte eine Strophe aus dem Französischen ins Englische; auch Freddie Fenders erfolgreiche Einspielung von »Before The Next Teardrop Falls« (1974) bot im spanischen Teil lediglich eine Übersetzung des englischsprachigen Originals von Duane Dee (1968); Johnnie Allans Swamp Pop-Versionen von »You Win Again« und »Today I Started Loving You Again« (beide 1980) übertragen wiederum einzelne Strophen bekannter Country-Nummern ins Französische (Hank Williams,

10 Während die Elvis-Nummer auf dem deutschen »Muss i denn, muss i denn zum Städtele hinaus « basierte, diente für Blondies Stück Randy \& The Rainbows (»Denise«, 1963) als Vorlage. Beides sind zweisprachig erweiterte Coverversionen, in denen Übersetzungen keine Rolle spielen. 
1952 / Merle Haggard, 1968). Leonard Cohens »The Partisan« (1969) beruht auf der englischen Nachdichtung eines französischen Liedes über den Widerstand gegen die Nazis, belässt aber eine Strophe in der Sprache des Originals und verdeutlicht so den Bezug zur französischen Résistance. Selten sind dagegen umfassende Nachdichtungen der Vorlage wie bei »The Girl From Ipanema« (Stan Getz/João Gilberto, 1964). ${ }^{11}$

Unabhängig von Coverversionen nimmt das Moment der Übersetzung generell einen wichtigen Stellenwert ein, auch in originär zweisprachigen Songs. Bei »Michelle« beginnt das Lied direkt mit einer Übersetzung, bei »Undestructable« der New Yorker Gipsy-Punk-Band Gogol Bordello kommt sie beispielsweise erst am Ende, wenn der aus der Ukraine stammende Sänger und Songwriter die vorangegangen englischen Textpassagen in einer freien Übersetzung auf Russisch wiederholt:

»How many... [darkest moments and traps still lay ahead of us?] - Undestructable

How many final frontiers with maybe no victory laps? - Undestructable

But if you stand on path of sacred art and stuck it out through thick and thin

God knows you become one

With undestructable

Ох сколько мгновений чёрных, Невозможных

Нам всем предстоит преодолеть. - (Undestructable)

Ох сколько пиров победных впереди,

Но всё равно нам не успеть. - (Undestructable)

Но если, если сокровенный путь один

Ты будешь несокрушим,

Несокрушим!«

(Gogol Bordello: »Undestructable«, 2005). ${ }^{12}$

Die übersetzende Funktion ist ein Grundmuster, das sich immer wieder in zweisprachigen Songs findet. Sie war schon in traditionellen Liedern vertreten, taucht in der neueren populären Musik indes ungleich häufiger auf. Ihre Erscheinungsweise ist recht unterschiedlich. Nur vereinzelt wird der gesamte Liedtext eins zu eins in die zweite Sprache übersetzt, wie bei Clifton

11 Bei diesem Titel ist wiederum bemerkenswert, dass sich die zweisprachige Version - mit Gesang von João und Astrud Gilberto - nur auf der Langspielplatte Getz/Gilberto (Verve 1964) findet, während die international bekannt gewordene Singleversion des Songs (mit Astrud Gilberto) nur die englische Textfassung enthält.

12 Die Aufnahme inszeniert einen quasi improvisatorischen Gestus dieser Übersetzung, der sich auch darin spiegelt, dass die russische Schlusspassage in der Bookletversion des Songtextes nicht berücksichtigt wird. 
Cheniers »Zydeco Two Step« (1980) oder Gordon Lightfoots »Nous Vivons Ensemble « (1971) - wobei im Falle Lightfoots diese egalitäre Form auch mit dem Inhalt und sozialen Anliegen des Liedes unmittelbar korrespondiert. Häufig wird nur eine Strophe in die zweite Sprache übertragen und mit weiteren (nicht übersetzten) Strophen kombiniert. ${ }^{13}$ Oder es wird nur der Text des Refrains in beiden Sprachen gesungen (Pete Seeger: "Somos El Barco 1985). Eine speziellere Form, die sich ebenfalls gelegentlich findet, sind kurze Einsprengsel von zwei bis drei Versen, die in einem ansonsten sprachlich homogenen Text eine kurze Passage in anderer Sprache wiederholen, etwa bei Rush: „Circumstances « (1978) oder Lady Gaga: »Bad Romance« (2009). Auch Interpretationsformen sind in diesem Zusammenhang strukturbildend, wenn die Übersetzung nicht mehr im gesungenen Part, sondern als gesprochener Text Teil der Aufnahme wird, wie bei Miriam Makeba: »Pata Pata« (1967), Visage: »Fade To Grey« (1980) oder Metric: »Poster Of A Girl« (2005).

Zur formalen Gestaltung zweisprachiger Songs lassen sich nur in begrenztem Rahmen Aussagen machen. Es gibt zwar einige Muster, die immer wieder Verwendung finden, aber aufs Ganze gesehen entzieht sich die Vielfalt der Erscheinungsformen einer systematischen Rubrizierung. Ein gängiges Schema zweisprachiger Lieder ist die Aufteilung der Sprachen zwischen Strophe und Refrain, wobei dieses Modell auch leicht variiert oder von anderen Elementen überlagert werden kann. Andere klar strukturierte Schemata wie der regelmäßige Sprachwechsel von Strophe zu Strophe - so Clifton Chenier: »Eh, Petite Fille« (1965) oder Altan: »Ta Mo Chleamhnas A Dheanamh $(1987)$ - und die Abfolge der Sprachen nacheinander (in zwei Teilen des Songs) - so Gorky's Zygotic Mynci: »Patio Song« (1996) oder Xavier Naidoo: »Ich kenne nichts« (2002) - sind selten. Solche schablonenhaften Verfahrensweisen markieren als Orientierungspunkte eher eine Tendenz, die nach Bedarf modifiziert wird. ${ }^{14}$ Ganz selten ist der regelmäßige Wechsel der Sprachen von Vers zu Vers. ${ }^{15}$ Gilles Vigneault griff beispielsweise mit »| Went To The Market « (1976) ein traditionelles bilinguales Lied auf, das diese Form aufweist, um damit auf humoristische Weise die unglei-

13 Z.B. Wayne Toups \& Zydecajun: "Sweet Joline« und »Two-Step Mamou« (beide 1989), Sandi Patty: »Via Dolorosa (1984), Rufus Wainwright: »Rebel Prince« (2001).

14 Freiere Verfahrensweisen dazu etwa bei Texas Tornados: "Soy de San Luis" (1990) und Gerardo Mejía: »Rico Suave (1991) bzw. Daniel Lanois: »Under A Stormy Sky« (1989) und Sublime »Caress Me Down« (1996).

15 Auch Travis' »Last Laugh Of The Laughter « (1999) ist in diesem Kontext eher ein Grenzfall, denn die beständig eingestreuten französischen Textelemente fungieren hier eher als Appendix zum jeweiligen englischen Vers. 
che Behandlung der (französischen) Minorität (und Sprache) in Kanada kritisch zu thematisieren.

Auch in anderen Fällen korrespondieren solche Eins-zu-Eins-Strukturen mit dem politischen oder sozialen Anliegen eines Songs - sei es im Sinne des demonstrativen Schulterschlusses gegen Fremdenfeindlichkeit und Rassismus oder des symbolischen Miteinanders separater Sprach- und Lebenswelten: Die beiden kanadischen Singer/Songwriter Dany Bédar und Joel Kroeker teilen sich in ihrem gemeinsamen Titel »Déjà Vu« (2007) paritätisch Strophen und Refrain (dort wechseln sie von Vers zu Vers), jeder Musiker singt in seiner Sprache, aber dies machen sie bewusst gemeinsam. Das dazu produzierte Video unterstreicht in seinen Bildern die Gespaltenheit der anglound frankophonen Lebenswelten in Kanada, die mit diesem Song wiederum überbrückt werden soll. Die umgekehrte formale Variante verwenden JeanJacques Goldman und Michael Jones in ihrem - gegen das damalige Erstarken der rechtsextremen Front National gerichteten - Song »Je te donne « (1985): Hier wechseln die Sprachen innerhalb der Strophen von Vers zu Vers, während der Refrain von beiden auf Französisch gesungen wird - auch hier dienen die formalen Strukturen als Abbild und Symbol der intendierten politischen Botschaft, die darauf zielt, Einflüsse aus unterschiedlichen Kulturen als Bereicherung und nicht als Gefahr zu begreifen.

Ausgeglichene Sprachproportionen in bilingualen Songs sind insgesamt in der Minderzahl. Überwiegend werden die Lieder so strukturiert, dass die zweite Sprache nur partiell Verwendung findet. Bei Melanies »What Have They Done To My Song Ma« (1970) ist es beispielsweise nur eine Strophe, die in der anderen Sprache gesungen wird. ${ }^{16}$ Oder es sind vergleichsweise kurze Einsprengsel, die freilich an exponierten Stellen des Songs eingebaut werden. Oft sind das nur zwei bis drei anderssprachige Verse, wie bei »Psycho Killer« (Talking Heads, 1977), »A tout le monde« (Megadeth, 1995) oder »Reflektor« (Arcade Fire, 2013). Es können auch Liedzitate sein, etwa bei Sophie Hunger: »Rise And Fall« (2008). ${ }^{17}$ Häufig sind es sogar eher Sprachsplitter, die aber durch die Art, wie sie verwendet werden, ein besonderes Gewicht erhalten - sei es im Refrain, aber auch zu Beginn oder am Schluss eines Songs. ${ }^{18}$ Bisweilen erscheint eine andere Sprache auch nur über

16 Ebenso Kimya Dawson \& Antsy Pants: »Tree Hugger« (2007), Arcade Fire: »Joan Of Arc « (2013) oder Chieftains: »Jimmy, Mó Mhíle Stór« (1999).

17 In diesen Song ist das »Guggisberglied« eingebaut, eines der prominentesten Schweizer Volkslieder.

18 Etwa R.E.M.: »Talk About The Passion« (1983), Yes: »Ritual (Nous sommes du Soleil)《 (Tales From Topographic Oceans, 1974), Franz Ferdinand: »Darts Of Pleasure « (2003), Sophie Hunger: »Monday's Ghost « (2008), oder - im Kontext des Refrains - Beatles: »Across The Universe« (siehe Anm. 5), Paolo Conte: 
Backing Vocals (Peter Gabriel: »Games Without Frontiers«, 1980) oder durch beigemischte Stimmen (Madonna: »Isaac «, 2005). Eine andere Verwendungsform der zweiten Sprache ist ihr Einsatz als Spiegelbild einer kommunikativen Szene (mit einem anderssprachigen Gegenüber). Schon bei »Michelle können die französischen Verse nicht nur im Sinne einer nationalen Verortung der Angebeteten verstanden werden, sondern auch als Ansprache an sie. Ebenso richten sich in »Angelina (Louis Prima, 1944/1957) die italienischen Wendungen direkt an die im Song verehrte Kellnerin in einer Pizzeria. Bei Bill Wymans »(Si Si) Je Suis Un Rock Star« (1981) dient der Wechsel zur französischen Rede dem englischen »rock star« wiederum als Vehikel zur Verständigung mit einer brasilianischen Schönheit. Umgekehrt ist es die Stimme der geliebten, fremden Frau, die sich in »The Ballad Of Cable Hogue (Calexico, 2000) in der anderen Sprache artikuliert. Ähnlich sind die Sprachrollen bei Arcade Fires »Joan Of Arc « (2013) verteilt, wenn die Stimme der Jeanne d'Arc auf Französisch erklingt. Dieses Prinzip findet auch bei sehr kurzen Texteinlagen Verwendung. ${ }^{19}$

In den letzten dreißig Jahren hat sich das Erscheinungsbild zweisprachiger Songs vor allem durch jene Musiker stark verändert, die solche Stücke ganz bewusst als Form und Ausdruck ihres ästhetischen Konzepts begreifen und Mehrsprachigkeit auf sehr vielfältige Weise in ihren Songs nutzen. Bei innen spielt wiederum das unmittelbare Code-Switching, der Sprachwechsel in seinen unterschiedlichsten Facetten, eine zentrale Rolle:

»Madame n'est pas

une Fräulein Schulz toute ordinaire,

Madame sait faire, pour toutes erreurs, sa vie...

und sonst so gut wie gar nichts.

[...]

Madame verspürt

ein großes Maß an `moi j'existe $<. .$.

Sie spürt nur sich,

das ist ihr Ich... und sonst,

und sonst so gut wie gar nichts«

(17 Hippies: »Madame«, 2007).

»Via con me (It's Wonderful)《 (1981), Beck: »Loser« (1994), Joe Cocker: »N'oubliez Jamais« (1997), Mano Negra: »Soledad« (1989), Silly Wizard: »Fhear A Bhata« (1978).

19 Beispielsweise bei Bob Dylan: »Black Diamond Bay« (Desire, 1976): »But the dealer says, >Attendez-vous, s'il vous plait - [...] she's out on the balcony, where a stranger tells her $>$ My darling, je vous aime beaucoup $\bullet . «$ 
Auch die direkte Thematisierung von Mehrsprachigkeit in Songs ist meist in solchem Kontext angesiedelt:

- Cypress Hill: »Latin Lingo« (1991)

- Mellow Man Ace: »The Brother With Two Tongues« (1992)

- Freundeskreis: »Esperanto« (1999)

- Daniel Kahn \& Painted Bird: »Broken Tongue« (2006)

- La Caravane Passe: »Perdu ta langue« (2010)

Mehrsprachigkeit, Code-Switching und bilinguale Songs stehen bei solchen Musikern häufig in einem Repertoirekontext, der sich nicht auf zwei Sprachen beschränkt. Gruppen wie die 17 Hippies, Mano Negra oder Gogol Bordello verwenden grundsätzlich mehrere Sprachen und dementsprechend finden sich unter ihren Songs auch unterschiedliche bilinguale Sprachkombinationen: bei Mano Negra beispielsweise mit Französisch, Spanisch und Englisch, bei Gogol Bordello Englisch, Russisch und Spanisch, bei RotFront Deutsch, Russisch, Ungarisch und Englisch. ${ }^{20}$

Solche Verwendung von Mehrsprachigkeit geht meist mit Ansätzen einher, auch musikalisch Sparten und Stile zu überschreiten und zu verbinden. Zweisprachige Songs werden hier Teil eines ästhetischen Konzepts, das sich insgesamt dem Aufmischen von Grenzen verschrieben hat, gerade auch in musikalischer Hinsicht. Dabei sind die Gruppen in den unterschiedlichsten musikalischen Milieus unterwegs, neben Weltmusik-Fusion (17 Hippies), Gypsy-Punk (Gogol Bordello) und Patchanka-Mestizo-Rock (Mano Negra, Manu Chao), beispielsweise auch Reggae-Ska-Punk (Che Sudaka), Rock-HipHop-Global-Dancehall (RotFront Emigrantski Raggamuffin), Gypsy-SwingBalkan-Pop (La Caravane Passe), Folk-Latin-Rock (Rupa \& The April Fishes), Pop-Volksmusik-Melange (Les Reines Prochaines) oder Party-HipHop (Culcha Candela). Zweisprachige Songs sind in diesen Kontexten stets Ausdruck eines transkulturell angelegten ästhetischen Konzeptes, ihre Mehrsprachigkeit flankiert die musikalisch-pluralistische Offenheit der Arbeitsweisen, sie bringt deren grenzgängerische Ambitionen buchstäblich zur Sprache.

Diese multikulturell intendierten musikalisch-ästhetischen Konzepte haben das Erscheinungsbild zweisprachiger Songs in den letzten Jahrzehnten am nachhaltigsten verändert. Ihre impliziten sozialen Positionsbestimmungen stehen in Wechselwirkung mit den entsprechenden politischen Diskursen. Parallel dazu haben sich aber auch andere Formen transnationaler und

20 Daran anknüpfend entstehen auch Songs, in denen mehr als zwei Sprachen genutzt werden: Il Gran Teatro Amaro verwendete im Text von "Mein Schatz « (Hotel Brennessel, 1993) beispielsweise Italienisch, Deutsch, Französisch und Englisch. 
transkultureller Inszenierungen mittels zweisprachiger Songs im Musikbusiness etabliert, sei es als symbolische Inszenierung (etwa bei internationalen Sport-Events), aber auch als pures Vermarktungs-Szenario, wie schon bei David Bowies bilingualen Versionen von »Heroes« (1977). ${ }^{21}$ Auch bei Placebo war die zweisprachige Version »Protège Moi« (2003) nur eine Produktion für den französischen Markt. Ein Meister der internationalen Vermarktung mittels zweisprachiger Songversionen ist zweifellos Andrea Bocelli. Die Grundlage dafür sind gemeinsame Duo-Aufnahmen mit Sängerinnen aus anderen Ländern:

\begin{tabular}{|l|l|l|}
\hline Andrea Bocelli & & \\
\hline $\begin{array}{l}\text { \& Judy Weiss } \\
\text { \& Hélène Ségara } \\
\text { \& Marta Sanchez } \\
\text { \& Sandy }\end{array}$ & »Vivo per lei« (1997) & $\begin{array}{l}\text { ital. / deutsch } \\
\text { ital. / franz. } \\
\text { ital. / span. } \\
\text { ital. / portug. }\end{array}$ \\
\hline \& Sarah Brightman & »Time To Say Goodbye (Con te partirò)« (1996) & engl. / ital. \\
\hline \& Celine Dion & »The Prayer« (1999) & ital. / engl. \\
\hline \& Dulce Pontes & »O Mar e tu« (1999) & ital. / portug. \\
\hline \& Marco Borsato & »Because We Believe« (2006) & ital. / holländ. \\
\hline
\end{tabular}

Tabelle 8: Andrea Bocelli

Viele andere Musiker nutzen ebenfalls dieses Duo-Modell, am liebsten in Kombination mit prominenten Namen, so auch Nena \& Kim Wilde mit »Anyplace, Anywhere, Anytime« (2002). Gegenüber früheren Vermarktungsstrategien mit Coverversionen eines Songs in verschiedenen Sprachen bieten solche zweisprachigen Aufnahmen den Vorteil, dass auch die ursprünglichen Interpreten in anderen Ländern und Märkten Präsenz zeigen können. DuoKonstellationen sind gleichfalls im Bereich der symbolischen Inszenierungen die mit Abstand häufigste Grundlage zweisprachiger Songs. Sie bilden hierfür das charakteristische Grundprinzip: sei es für musikalische Demonstrationen politischen Engagements oder für die Kreation eines Flairs von Welt bei internationalen Sportveranstaltungen (s. Tabelle 9).

Zweisprachige Songs bilden einen thematisch weit umfassenderen Komplex als dies auf den ersten Blick erscheinen mag. Festzuhalten bleibt, dass Bilingualität schon seit langer Zeit ein Ingrediens populärer Musik ist. Indes haben sich ihre Präsenz, ihre Funktionen und ihre Erscheinungsformen in den

21 Unter den verschiedenen Versionen des Songs, die Bowie damals auf den internationalen Markt brachte, waren neben den englischen auch zweisprachige Einspielungen (mit Deutsch und mit Französisch) vertreten - wobei die deutschenglische Aufnahme aufgrund ihrer Verwendung im Film Christiane F. - Wir Kinder vom Bahnhof Zoo (1981) damals recht bekannt wurde. 


\begin{tabular}{|c|c|}
\hline Udo Lindenberg \& Alla Pugacheva & »Wozu sind Kriege da?« (Radio Erewan, 1985) \\
\hline Holly Near \& Mercedes Sosa & »They Dance Alone« (Singer In The Storm, 1990) \\
\hline $\begin{array}{l}\text { Freddie Mercury \& Montserrat } \\
\text { Caballe }\end{array}$ & $\begin{array}{l}\text { »Barcelona« (1987) - Wiederveröffentlichungen } \\
\text { zur Olympiade } 1992 \text { sowie zum Champions League } \\
\text { Finale } 1999\end{array}$ \\
\hline Sting \& Mariza & $\begin{array}{l}\text { »A Thousand Years« (2004) - Olympische Spiele } \\
\text { Athen }\end{array}$ \\
\hline $\begin{array}{l}\text { Herbert Grönemeyer } \\
\text { feat. Amadou \& Mariam }\end{array}$ & $\begin{array}{l}\text { »Zeit, dass sich was dreht / Celebrate The Day / } \\
\text { Fetez cette journée« (2006) - Fußball-WM } 2006\end{array}$ \\
\hline $\begin{array}{l}\text { Pitbull feat. Jennifer Lopez \& } \\
\text { Claudia Leitte }\end{array}$ & »We Are One« (2014) - Fußball-WM 2014 \\
\hline
\end{tabular}

Tabelle 9: Symbolische Inszenierungen (Politik - Sport)

letzten drei Jahrzehnten stark verändert. Neu dabei ist, dass Mehrsprachigkeit Teil musikalisch-ästhetischer Konzepte wird. Vielfach ist dies damit verknüpft, dass Mitglieder entsprechender Bands aus unterschiedlichen Sprachkulturen kommen. Auch darüber hinaus gibt es bei zweisprachigen Songs häufig Bezüge zu biographischen Prägungen der jeweiligen Musiker. Vieles konnte in vorliegendem Beitrag nur angerissen werden, manche Gesichtspunkte müssen einstweilen gänzlich offen bleiben: Thematische Aspekte etwa wie jene Songs, die auf dem Vergnügen am Spiel mit unterschiedlichen Sprachen gründen, ${ }^{22}$ aber auch Stücke, in denen eine zweite Sprache im ironisch-abwertenden Sinne verwendet wird, ${ }^{23}$ ebenso das Terrain mehrsprachiger Songs beim Eurovision Song Contest ${ }^{24}$ oder in der internationalen Rapund HipHop-Musik, und letztlich auch die Rezeption zweisprachiger Songs, ihre Coverversionen und Parodien. ${ }^{25}$ Hinzu kommen andere geographische Blickwinkel, denn zweisprachige Songs haben einen globalen Geltungsbereich und es gibt sie selbstverständlich auch in der Pop- und Rockmusik von Regionen und Sprachkombinationen, von denen hier nicht die Rede war. Und natürlich bleiben auch viele Fragen zu konkreten Stücken offen: Warum wechselt Melanie in »What Have They Done To My Song Ma« eigentlich in einer Strophe ins Französische? Was sollen uns die deutschsprachigen Ein-

22 Seien es Songs wie Helge Schneiders »Sommer, Sonne, Kaktus!« (Sommer, Sonne, Kaktus!, 2013) oder Kraftwerks »Nummern « (Computerwelt, 1981).

23 In diesem Kontext wäre auch Rammstein: »Amerika« (Reise, Reise, 2004) zu erwähnen.

24 Bilinguale Songs beim Eurovision Song Contest sind ein weiteres, gleichermaßen aufschlussreiches wie vielschichtiges Feld der transnationalen Inszenierungen, das nicht nur wegen der zahlreichen Stücke, sondern auch aufgrund seines spezifischen Kontextes einer separaten Darstellung bedarf.

25 Solche satirischen Verarbeitungen können wiederum neue bilinguale Songs generieren, wie »Weird Al« Yankovics »Taco Grande« (Off The Deep End, 1992), eine Parodie auf »Rico Suave« (1991). 
sprengsel in Stücken Frank Zappas sagen ${ }^{26}$ Und was bedeutet in »Psycho Killer« der Talking Heads die französische Bridge und der Refrain: »Psycho Killer, qu'est-ce que c'est?«

»|'ve tried to explain, bei mir bist du schön / So kiss me, and say you understand«, so sangen die Andrews Sisters 1938 und mit ihnen viele andere. Zwei Generationen später hat sich manches verändert, bilinguale Musiker artikulieren sich weitaus selbstbewusster, finden Zweisprachigkeit hip und gleichermaßen sexy: »All I want is a bilingual girl« verkündet die New Yorker Band Yerba Buena in ihrem - selbstredend zweisprachigen - Song »Bilingual Girl« (Island Life, 2005), denn schließlich müsse doch jeder verstehen: »Two tongues are better than one .

\section{Literatur}

Helms, Dietrich (2014). »History? My story! Ein Plädoyer für das Ich in Pop-Geschichte. "In: Geschichte wird gemacht. Zur Historiographie populärer Musik. Hg. v. Dietrich Helms und Thomas Phleps (= Beiträge zur Popularmusikforschung 40). Bielefeld: transcript, S. 115-127.

John, Eckhard (2015). »Mit gespaltener Zunge. Zweisprachige Lieder als Forschungsfeld. "In: Musik in der »Lebenswelt «. Immaterielle Dimensionen der Musik-Kultur von Deutschen in (und aus) den südosteuropäischen Siedlungsgebieten. Hg. v. Erik Fischer et al. Stuttgart (im Druck).

Lebrun, Barbara (2009). Protest Music in France. Production, Identity and Audiences. Farnham: Ashgate [zu Mano Negra s. S. 89-106].

Miles, Barry (1998). Paul McCartney. Many Years From Now. Reinbek bei Hamburg: Rowohlt.

Schillmöller, Mathias (2012). » Je veux devenir ton étrangère...< - Berlin Style mit französischer Note bei den 17 Hippies. « In: Lied und populäre Kultur 57, S. 347360.

\section{Diskographie}

Gogol Bordello (2005). „Undestructable.« Auf: Gypsy Punks. Underdog World Strike. SideOneDummy Records SD1271.

17 Hippies (2007). »Madame. « Auf: Heimlich. Hipster-Records HIP 012.

Spliff (1982). »Carbonara.« Auf: 85555. CBS 85555.

The Andrews Sisters (1938). »Bei bist du schön. « Auf: The Andrews Sisters. Lotus Records LOP 14101 (1984).

The Beatles (1965). »Michelle. « Auf: Rubber Soul. Parlophone PMC 1267.

The Clash (1979). »Spanish Bombs. « Auf: London Calling. CBS 88478.

26 Etwa Frank Zappa: »Sofa No. 2« (One Size Fits All, 1975), "Stick It Out« (Joe's Garage, Act II, 1979), »Once Upon A Time« (You Can't Do That On Stage Anymore Vol. I, 1988). 


\begin{abstract}
The existence of bilingual songs has played no significant role in popular music memory so far as they are often not remembered as being bilingual. Nevertheless, bilingualism has been an ingredient of popular music for a long time. However, the presence of bilingual texts, their functions, and their manifestations have strongly changed during the last 30 years. It is a new development that multilingualism becomes a part of musical-aesthetic concepts. Direct code-switching in many different facets plays a central role in these songs. Such a use of multilingualism is usually associated with approaches that also cross and connect genres and styles musically. Here, bilingual songs become a part of artistic attempts that are committed to challenging boundaries, especially in musical terms. These multiculturally orientated concepts have changed the appearance of bilingual songs in recent decades with the most lasting effect. Their implicit social intentions interact with corresponding political discourses. At the same time, however, other forms of transnational and transcultural productions have been established in the music business by means of bilingual songs, whether for symbolic production (as in international sports events) or purely for marketing means. Moreover, a long-term continuity of certain features of multilingualism in popular songs can also be observed, particularly in terms of their coloristic functions or usages of translations.
\end{abstract}

